\title{
ARCADE 2 OBSERVATIONS OF GALACTIC RADIO EMISSION
}

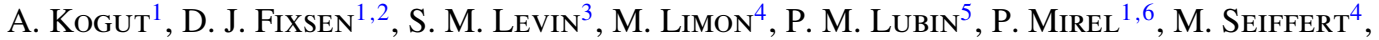 \\ J. Singal ${ }^{7}$, T. Villela ${ }^{8}$, E. Wollack ${ }^{1}$, And C. A. WuensChe ${ }^{8}$ \\ ${ }^{1}$ Code 665, Goddard Space Flight Center, Greenbelt, MD 20771, USA; Alan.J.Kogut@nasa.gov \\ ${ }^{2}$ Department of Physics and Astronomy, University of Maryland, Code 665, Goddard Space Flight Center, Greenbelt, MD 20771, USA \\ ${ }^{3}$ Jet Propulsion Laboratory, California Institute of Technology, 4800 Oak Grove Drive, Pasadena, CA 91109, USA \\ ${ }^{4}$ Columbia Astrophysics Laboratory, 550W 120th Street, Mail Code 5247, New York, NY 10027-6902, USA \\ ${ }^{5}$ Department of Physics, Broida Hall, Mail Code 9530, University of California, Santa Barbara, CA 93106, USA \\ ${ }^{6}$ Wyle Information Systems, Code 665, Goddard Space Flight Center, Greenbelt, MD 20771, USA \\ ${ }^{7}$ Kavli Institute for Particle Astrophysics and Cosmology, SLAC National Accelerator Laboratory, Menlo Park, CA 94025, USA \\ ${ }^{8}$ Instituto Nacional de Pesquisas Espaciais, Divisão de Astrofísica, Caixa Postal 515, 12245-970, São José dos Campos, SP, Brazil \\ Received 2011 February 18; accepted 2011 March 28; published 2011 May 17
}

\begin{abstract}
We use absolutely calibrated data from the ARCADE 2 flight in 2006 July to model Galactic emission at frequencies 3, 8, and $10 \mathrm{GHz}$. The spatial structure in the data is consistent with a superposition of free-free and synchrotron emission. Emission with spatial morphology traced by the Haslam $408 \mathrm{MHz}$ survey has spectral index $\beta_{\text {synch }}=-2.5 \pm 0.1$, with free-free emission contributing $0.10 \pm 0.01$ of the total Galactic plane emission in the lowest ARCADE 2 band at $3.15 \mathrm{GHz}$. We estimate the total Galactic emission toward the polar caps using either a simple plane-parallel model with csc $|b|$ dependence or a model of high-latitude radio emission traced by the $C O B E$ /FIRAS map of $\mathrm{C}$ II emission. Both methods are consistent with a single power law over the frequency range $22 \mathrm{MHz}$ to $10 \mathrm{GHz}$, with total Galactic emission toward the north polar cap $T_{\mathrm{Gal}}=10.12 \pm 0.90 \mathrm{~K}$ and spectral index $\beta=-2.55 \pm 0.03$ at reference frequency $0.31 \mathrm{GHz}$. Emission associated with the plane-parallel structure accounts for only $30 \%$ of the observed high-latitude sky temperature, with the residual in either a Galactic halo or an isotropic extragalactic background. The well-calibrated ARCADE 2 maps provide a new test for spinning dust emission, based on the integrated intensity of emission from the Galactic plane instead of cross-correlations with the thermal dust spatial morphology. The Galactic plane intensity measured by ARCADE 2 is fainter than predicted by models without spinning dust and is consistent with spinning dust contributing $0.4 \pm 0.1$ of the Galactic plane emission at $23 \mathrm{GHz}$.
\end{abstract}

Key words: cosmic background radiation - radiation mechanisms: non-thermal - radio continuum: ISM

Online-only material: color figures

\section{INTRODUCTION}

The cosmic microwave background (CMB) is a valuable probe of physical conditions in the early universe. Its frequency spectrum records the history of energy transfer between the evolving matter and radiation fields to constrain the energetics of the early universe. We view the CMB through diffuse emission from the interstellar medium. At centimeter wavelengths, the dominant contributions are from synchrotron emission originating from electrons accelerated in the Galactic magnetic field, and free-free emission (thermal bremsstrahlung) from electron-ion collisions. Thermal emission from interstellar dust is negligible, but electric dipole emission from a population of small, rapidly rotating dust grains could contribute a substantial fraction of the total Galactic emission at wavelengths near $1 \mathrm{~cm}$ (Draine \& Lazarian 1998; Dobler \& Finkbeiner 2008; Miville-Deschênes et al. 2008). Absolute measurements of the sky temperature at centimeter wavelengths measure diffuse emission to separate Galactic from primordial emission and provide information on physical processes in the interstellar medium.

The Absolute Radiometer for Cosmology, Astrophysics, and Diffuse Emission (ARCADE) is an instrument to measure the absolute temperature of the sky in search of distortions from a blackbody spectrum. ARCADE operates at centimeter wavelengths between full-sky surveys at radio frequencies below $3 \mathrm{GHz}$ and the Far Infrared Absolute Spectrophotometer (FIRAS) survey at frequencies above $60 \mathrm{GHz}$. It consists of a set of cryogenic radiometers observing at $37 \mathrm{~km}$ altitude from a balloon payload. Each radiometer uses a double-nulled design, measuring the temperature difference between a corrugated horn antenna and an internal reference as the antenna alternately views the sky or a full-aperture blackbody calibrator. The internal reference can be adjusted to null the antenna-reference signal difference, while the calibrator temperature can be independently adjusted to null the sky-calibrator signal difference. ARCADE thus measures small shifts about a precise blackbody, greatly reducing dependence on instrument calibration and stability. The calibrator, antennas, internal reference, and radiometer front-end amplifiers are mounted within a large liquid helium Dewar and are maintained near thermal equilibrium with the CMB. Boiloff helium vapor, vented through the aperture, forms a barrier between the instrument and the atmosphere to allow operation in full cryogenic mode with no windows between the optics and the sky.

A two-channel prototype (ARCADE 1) flew in 2001 and again in 2003 , observing the sky at 10 and $30 \mathrm{GHz}$ to demonstrate the feasibility of cryogenic open-aperture optics (Kogut et al. 2004; Fixsen et al. 2004). A second-generation six-channel instrument (ARCADE 2) flew in 2005 and again in 2006. A motor failure in 2005 allowed only a single channel to view the sky (Singal et al. 2006). The 2006 flight successfully obtained observations at 3.3, 8.3, 10.2, 30, and $90 \mathrm{GHz}$. Data from the $2006 \mathrm{flight}$ are consistent with a blackbody CMB spectrum, but show a clear detection of an extragalactic radio background. This paper 


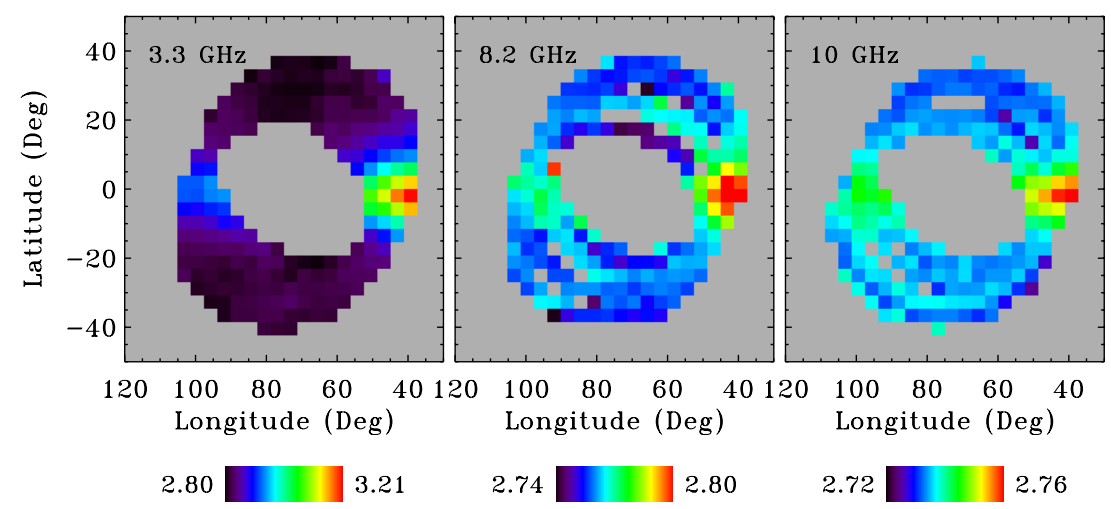

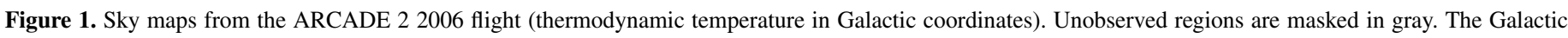
plane is clearly visible.

(A color version of this figure is available in the online journal.)

Table 1

Frequency Bands for Foreground Analysis

\begin{tabular}{ccc}
\hline \hline $\begin{array}{c}\text { Frequency } \\
(\mathrm{GHz})\end{array}$ & $\begin{array}{c}\text { Bandwidth } \\
(\mathrm{MHz})\end{array}$ & $\begin{array}{c}\text { White Noise } \\
\left(\mathrm{mK} \mathrm{s}^{1 / 2}\right)\end{array}$ \\
\hline 3.20 & 210 & 11.8 \\
3.41 & 220 & 10.1 \\
7.98 & 350 & 5.5 \\
8.33 & 350 & 5.2 \\
9.72 & 860 & 3.0 \\
10.49 & 860 & 3.0 \\
\hline
\end{tabular}

describes the analysis of Galactic emission from the 2006 flight. Companion papers describe the ARCADE 2 instrument (Singal et al. 2011), the calibration and sky temperature analysis (Fixsen et al. 2011), and the results from fits to potential distortions from a CMB blackbody spectrum (Seiffert et al. 2011).

\section{OBSERVATIONS AND SKY MAPS}

The ARCADE 2 instrument launched from the Columbia Scientific Balloon Facility in Palestine, TX, carrying a complement of seven Dicke-switched radiometers and 1800 liters of liquid helium. During ascent, the Dicke switch failed in one radiometer. The remaining radiometers began sky observations on 2006 July 22 at 05:08 UT, continuing through 08:11 UT just prior to flight termination.

Figure 1 shows the absolute sky temperatures binned by Galactic coordinates. Each radiometer views the sky through a corrugated conical feed horn, scaled with wavelength to produce a Gaussian beam with 11.6 full width at half-maximum (Singal et al. 2005). The beams point $30^{\circ}$ from the zenith and the entire payload spins at $0.6 \mathrm{rpm}$ so that the beams scan a circle of $60^{\circ}$ diameter centered on the zenith. The spin frequency is comparable to the $1 / f$ knee of the instrument noise, so that striping at the $\mathrm{mK}$ level is visible in the binned data. Each radiometer observed roughly $7 \%$ of the sky.

The measured sky temperatures are dominated by the CMB monopole. Galactic emission is clearly visible in the data at 3, 8, and $10 \mathrm{GHz}$. At higher frequencies, the smaller Galactic signal and higher instrument noise combine to prevent a clear detection of Galactic emission. Throughout this paper, we consider only data from the 3,8 , and $10 \mathrm{GHz}$ radiometers. Table 1 summarizes the in-flight performance of each radiometer. We compute the instrument noise by comparing the variance of the data within each sky bin to the number of observations in that bin. This value for the noise thus includes calibration as well as the effect of low-frequency striping.

The observed emission is a combination of CMB, synchrotron, and free-free emission. Synchrotron emission results from the acceleration of cosmic-ray electrons in the Galactic magnetic field. For a power-law distribution of electron energies $N(E) \propto E^{-p}$ propagating in a uniform magnetic field, the synchrotron emission is also a power law,

$$
T_{A}(v) \propto v^{-\beta_{s}}
$$

where $T_{A}$ is antenna temperature, $v$ is the radiation frequency, and $\beta_{s}=-(p+3) / 2$ (Rybicki \& Lightman 1979). Free-free emission is also a power law in antenna temperature, with spectral index $\beta_{\mathrm{ff}}=-2.15$.

We evaluate the observed spectral index in the ARCADE 2 sky maps by plotting the temperature observed at one frequency against the temperature of the same sky pixel observed at a different frequency. Since the ARCADE 2 absolute calibration is derived from comparison of sky data to a blackbody calibrator of known physical temperature, the sky solutions have units of thermodynamic temperature (Fixsen et al. 2011). In order to evaluate the spectral index, we convert the sky maps (Figure 1) from thermodynamic to antenna temperature

$$
T_{A}=\frac{x}{\exp (x)-1} T
$$

where $T$ is thermodynamic temperature, $x=h v / k T, h$ is Planck's constant, and $k$ is Boltzmann's constant. Figure 2 shows the resulting $T-T$ plots.

The same Galactic features are observed at each frequency. A linear fit to each plot yields spectral index $\beta=-2.43 \pm 0.03$ from 3.3 to $8.3 \mathrm{GHz}, \beta=-2.47 \pm 0.02$ from 3.3 to $10.3 \mathrm{GHz}$, and $\beta=-3.12 \pm 0.17$ from 8.3 to $10.3 \mathrm{GHz}$, where the quoted errors include the measurement uncertainties in both maps for each plot. The measured index is consistent with a superposition of synchrotron and free-free emission.

\section{SPATIAL STRUCTURE}

A search for distortions in the $\mathrm{CMB}$ spectrum requires correction of Galactic foreground emission. The ARCADE 2 data have only six frequency channels containing significant Galactic emission (Table 1) and cannot uniquely separate the observed emission into individual components (synchrotron, free-free, or other sources). We combine the ARCADE 2 data with other sky surveys in order to model foreground emission. 

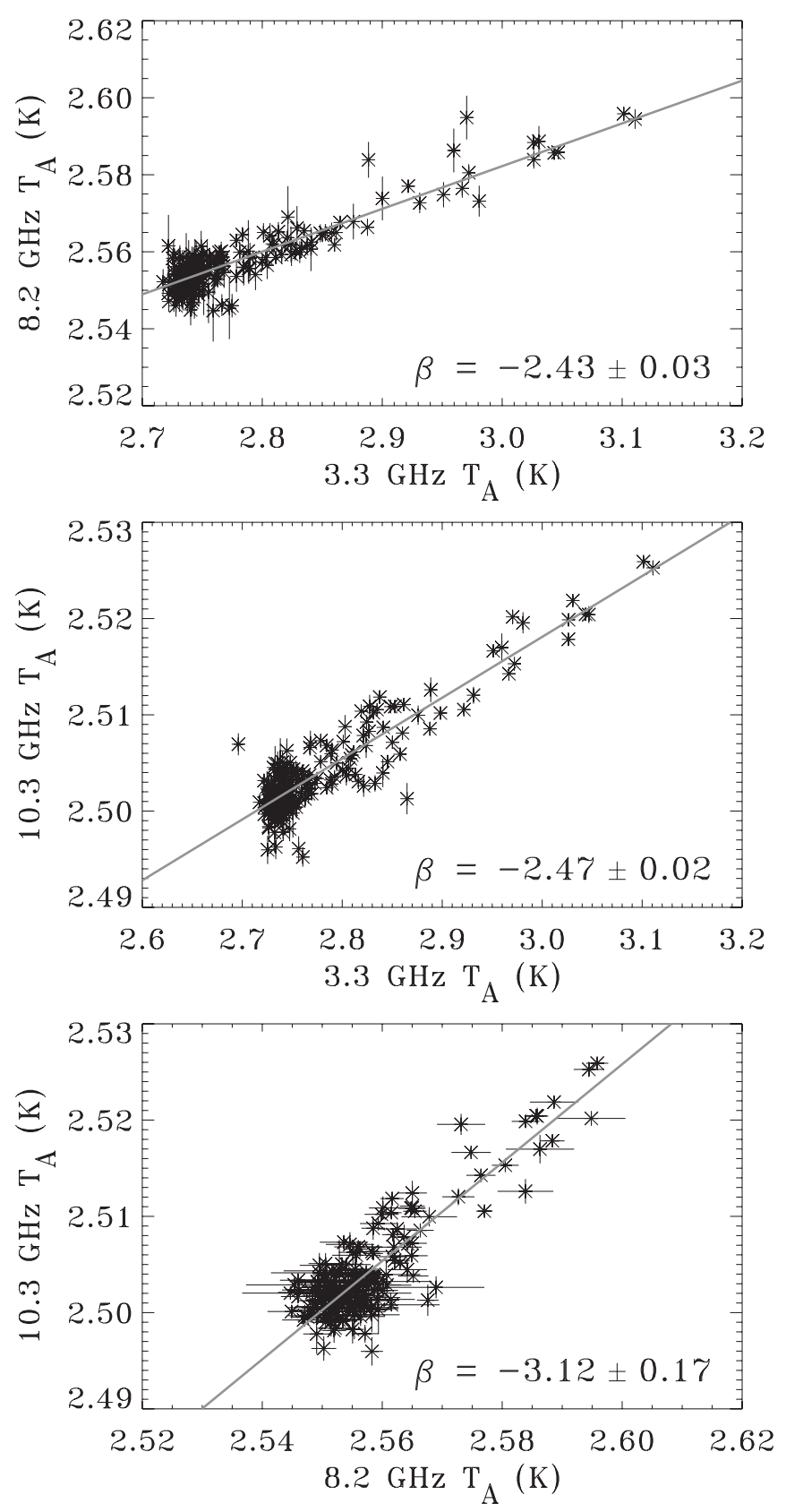

Figure 2. $T-T$ plots from the ARCADE 2 sky maps in units of antenna temperature. The best-fit spectral index (solid line) is consistent with a superposition of synchrotron and free-free emission.

A widely used technique describes the observed emission at each frequency $v$ as a linear combination of fixed "template" maps,

$$
T_{A}(v, p)=\sum_{i} \alpha_{i}(v) G_{i}(p)
$$

where $p$ is a pixel index and $G_{i}(p)$ are a set of sky maps tracing different components of the interstellar medium. The spectral dependence of the fitted coefficients $\alpha(v)$ can then be ascribed to the component traced by each template.

Several choices for template maps tracing free-free emission are available. Gold et al. (2009) use a maximum entropy algorithm to derive a map of free-free emission based on the Wilkinson Microwave Anisotropy Probe (WMAP) five-year data and a compilation of $\mathrm{H} \alpha$ emission (Finkbeiner 2003). Since the
Table 2

Coefficients for Spatial Templates

\begin{tabular}{ccc}
\hline \hline $\begin{array}{c}\text { Frequency } \\
(\mathrm{GHz})\end{array}$ & $\begin{array}{c}\alpha_{\mathrm{ff}} \\
\left(\mathrm{mK} \mathrm{nW}^{-1} \mathrm{~m}^{2} \mathrm{sr}\right)\end{array}$ & $\begin{array}{c}\alpha_{s} \\
(\mathrm{mK} / \mathrm{K})\end{array}$ \\
\hline 3.20 & $3.22 \pm 0.11$ & $2.02 \pm 0.05$ \\
3.41 & $3.06 \pm 0.09$ & $1.70 \pm 0.04$ \\
7.98 & $0.26 \pm 0.05$ & $0.24 \pm 0.02$ \\
8.33 & $0.28 \pm 0.05$ & $0.24 \pm 0.02$ \\
9.72 & $0.39 \pm 0.03$ & $0.04 \pm 0.01$ \\
10.49 & $0.37 \pm 0.03$ & $0.05 \pm 0.01$ \\
\hline
\end{tabular}

maximum entropy method (MEM) template is heavily weighted by the microwave data in the Galactic plane, it minimizes extinction effects in the $\mathrm{H} \alpha$ map. As alternatives to the maximum entropy template, we also use either a map of thermal dust emission (Finkbeiner et al. 1999, model 8) or the FIRAS map of C II emission at $158 \mu \mathrm{m}$ (Fixsen et al. 1999), neither of which is affected by extinction.

The choice of synchrotron template depends on the treatment of spatial variation in the synchrotron spectral index. To the extent that this variation can be neglected, the $408 \mathrm{MHz}$ survey (Haslam et al. 1981) provides a high signal-to-noise ratio tracer for synchrotron emission. Since the $408 \mathrm{MHz}$ survey contains both free-free and synchrotron emission, we correct it for free-free emission using the MEM free-free template scaled to $408 \mathrm{MHz}$ using a spectral index $\beta_{\mathrm{ff}}=-2.15$ so that to first order the template represents only synchrotron emission. We convolve both the synchrotron and free-free templates to match the ARCADE 11.6 beam width.

The ARCADE 2 data are dominated by the CMB monopole, even toward the Galactic plane. Both the Galactic and extragalactic signals also contain monopole terms, as do the synchrotron and free-free templates. We thus include a monopole template as a nuisance parameter and compute the template coefficients $\alpha_{i}(v)$ by minimizing

$$
\chi^{2}=\sum_{p} \frac{\left[T_{A}(p, v)-\alpha_{0}(v)-\alpha_{\mathrm{ff}}(v) G_{\mathrm{ff}}(p)-\alpha_{\mathrm{s}}(\nu) G_{\mathrm{s}}(p)\right]^{2}}{\sigma(p, v)^{2}},
$$

where $\alpha_{0}, \alpha_{\mathrm{ff}}$, and $\alpha_{s}$ are the coefficients for the monopole, free-free, and synchrotron templates, respectively, and $\sigma(p, v)$ is the instrument noise in each pixel and frequency channel.

The spatial structure in the ARCADE 2 data may be described as a superposition of emission traced by the Haslam $408 \mathrm{MHz}$ survey and the FIRAS map of C II emission. Table 2 shows the fitted coefficients for the best-fit template combination. Fitting the coefficients from each template to a power law in frequency yields spectral indices $-2.5 \pm 0.1$ for emission traced by the $408 \mathrm{MHz}$ survey and $-2.0 \pm 0.1$ for emission traced by the C II map.

The best template model uses the $408 \mathrm{MHz}$ survey to trace synchrotron emission and the FIRAS map of C II line emission to trace free-free emission. We have repeated the template fits using different tracers for synchrotron or free-free emission, obtaining broadly similar results. In all cases, the best choice for free-free template is either the $C_{\text {II }}$ map or the thermal dust map. We note that spatial structure in the ARCADE 2 maps is dominated by the Galactic plane, where extinction corrections for free-free maps derived from $\mathrm{H} \alpha$ emission are worst. The ratio of emission traced by the $\mathrm{C}$ II free-free template to emission traced by the $408 \mathrm{MHz}$ synchrotron template is $\left\langle T_{\mathrm{ff}} / T_{\text {synch }}\right\rangle=0.16 \pm 0.01$ evaluated for latitudes $|b|<10^{\circ}$ 


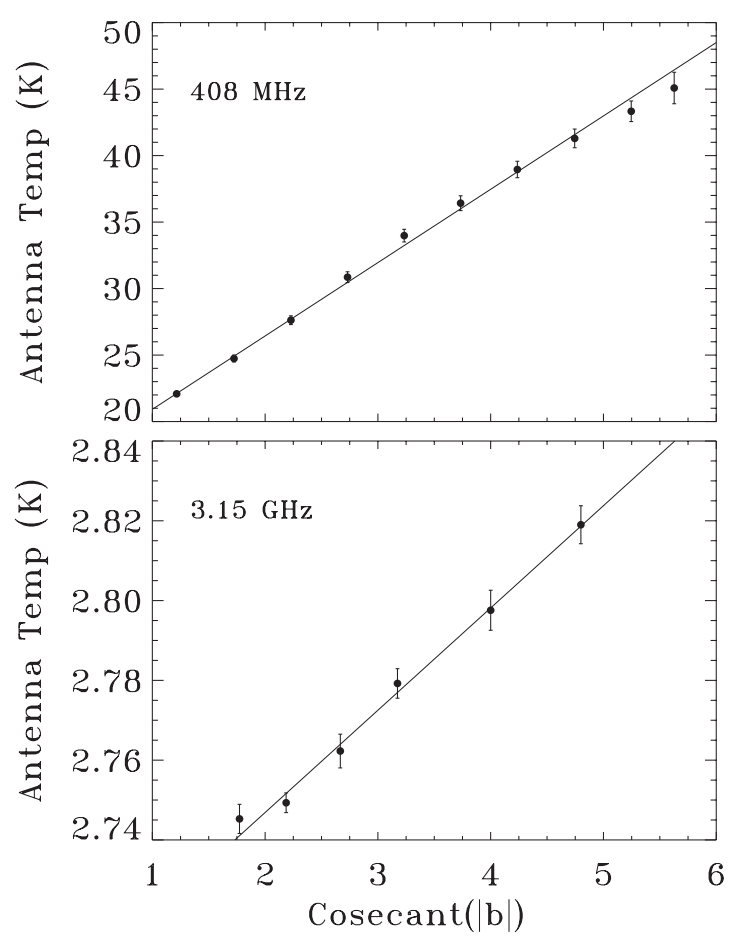

Figure 3. Temperature of the $408 \mathrm{MHz}$ survey (top) and ARCADE $3.15 \mathrm{GHz}$ channel (bottom) binned by $\csc (b)$ for latitudes $b>10^{\circ}$ (Northern hemisphere). Sky temperatures include the CMB and any extragalactic background. The scatter about the best-fit line results from the higher-order spatial structure in the maps. For clarity, the plotted uncertainties have been inflated by a factor of 20 (408 MHz survey) or 5 (ARCADE); the actual statistical uncertainties for the mean of each bin are smaller than the symbol size. The data are consistent with a plane-parallel model whose measured slope provides an estimate of the Galactic emission at the poles.

in the lowest ARCADE 2 channel at $3.15 \mathrm{GHz}$. The ratio of free-free emission to the total Galactic plane emission is $\left\langle T_{\mathrm{ff}} / T_{\text {total }}\right\rangle=0.10 \pm 0.01$.

\section{GALACTIC POLAR CAP TEMPERATURE}

The sky temperature measured by ARCADE 2 includes contributions from Galactic, extragalactic, and cosmic sources. The 2.7 K CMB dominates the measured temperatures. Galactic emission is dominated by synchrotron emission, with a smaller contribution from free-free sources. The integrated contribution of similar synchrotron and free-free emission in external galaxies constitutes an isotropic extragalactic radio background. Accurate measurement of the extragalactic or cosmic backgrounds requires reliable determination of both the spatial structure and the zero level of the Galactic emission.

Although the CMB may be distinguished from Galactic or extragalactic radio emission by the different frequency dependences, spectral fitting alone cannot distinguish Galactic emission from an extragalactic component of similar spectral behavior. The template model above reproduces the spatial structure of the observed Galactic emission, but is insensitive to emission described by an additive constant in each map. If the total Galactic brightness were determined along some fiducial line of sight, we could add a monopole term to the template model to match the total Galactic brightness along that line of sight. The adjusted model would then characterize Galactic emission along all other lines of sight.

The north and south polar caps (latitude $|b|>75^{\circ}$ ) provide convenient reference lines of sight. A commonly used method

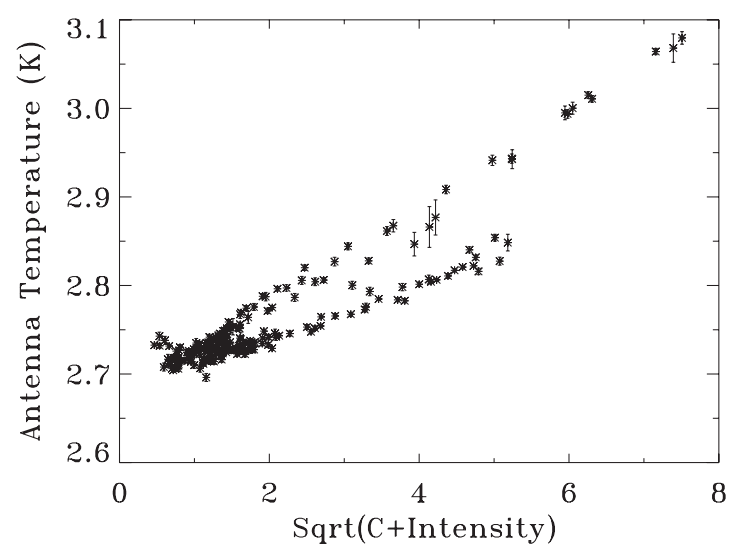

Figure 4. Correlation between the ARCADE $3.15 \mathrm{GHz}$ intensity and the $\mathrm{C}_{\mathrm{II}}$ $158 \mu \mathrm{m}$ atomic line in units $\mathrm{nW} \mathrm{m} \mathrm{m}^{-2} \mathrm{sr}^{-1}$. The data clearly divide into two regions each with radio intensity proportional to the square root of the $\mathrm{CII}$ intensity. The upper track originates from pixels at longitude $l \sim 45^{\circ}$ (closer to the Galactic center), while the lower track originates from pixels at longitude $l \sim 95^{\circ}$ (closer to the Cygnus region).

to estimate total Galactic brightness toward the poles models the Galaxy as a simple plane-parallel structure and fits the spatial distribution of the radio continuum at Galactic latitude $|b|>10^{\circ}$ to the form

$$
T_{A}(v, p)=c(v)+T_{G}(v) \csc |b|,
$$

where $b$ is the Galactic latitude of each pixel $p$ and the constant $c(v)$ accounts for the contribution from the CMB or extragalactic backgrounds at each frequency $v$. Figure 3 shows typical results for the northern hemisphere, both for the lowest frequency ARCADE 2 channel and the $408 \mathrm{MHz}$ survey. The high-latitude structure of the radio sky is consistent with a plane-parallel model, whose slope defines the antenna temperature $T_{G}(v)$ of Galactic emission toward the Galactic poles.

The simple cosecant model traces radio emission associated with the plane-parallel structure of the Galaxy, but averages over significant longitudinal structure and is insensitive to emission from a halo or similar extended high-latitude structure. As a cross-check, we correlate maps of the radio sky with maps of line emission originating within the Galaxy to estimate the radio intensity corresponding to the measured Galactic line brightness along high-latitude lines of sight. Several lines could be used to trace Galactic microwave emission. $\mathrm{H} \alpha$ emission from 3 to 2 transition in neutral atomic hydrogen has been mapped over the full sky and is a well-established tracer of free-free emission. However, $\mathrm{H} \alpha$ emission predominantly traces free-free emission from the warm ionized medium and may not accurately trace emission from the brighter synchrotron component. In addition, $\mathrm{H} \alpha$ emission suffers from dust extinction in the Galactic plane making it less reliable in regions where the Galactic radio emission is brightest. The $21 \mathrm{~cm}$ fine-structure line from neutral hydrogen has also been mapped over the full sky. However, since $\mathrm{H}$ I emission originates from the neutral component of the interstellar medium, it is unlikely to trace microwave emission from ionized regions. The $\mathrm{C}_{\mathrm{II}}$ line at $158 \mu \mathrm{m}$ wavelength from singly ionized carbon can be used to trace diffuse radio emission. It is optically thin even in the plane, does not suffer from dust extinction, and is an important cooling mechanism for the diffuse interstellar medium. It has been mapped over the full sky by the $C O B E /$ FIRAS instrument (Fixsen et al. 1999).

Figure 4 shows the correlation between the ARCADE $3.15 \mathrm{GHz}$ map and the $\mathrm{C}_{\text {II }} 158 \mu \mathrm{m}$ map. The $\mathrm{C}_{\text {II }}$ map 
Table 3

Radio/C II Correlation Slope

\begin{tabular}{lccc}
\hline \hline \multirow{2}{*}{$\begin{array}{c}\text { Frequency } \\
(\mathrm{GHz})\end{array}$} & Component 1 & Component 2 & Mean \\
\cline { 2 - 4 } 0.022 & $(1.12 \pm 0.06) \times 10^{4}$ & $(0.74 \pm 0.02) \times 10^{4}$ & $(0.93 \pm 0.19) \times 10^{4}$ \\
0.045 & $(2.74 \pm 0.07) \times 10^{3}$ & $(1.56 \pm 0.03) \times 10^{3}$ & $(2.15 \pm 0.63) \times 10^{3}$ \\
0.408 & $18.1 \pm 0.3$ & $8.5 \pm 0.1$ & $13.3 \pm 5.0$ \\
1.420 & $(5.11 \pm 0.08) \times 10^{-1}$ & $(2.94 \pm 0.04) \times 10^{-1}$ & $(4.02 \pm 1.10) \times 10^{-1}$ \\
3.20 & $(5.63 \pm 0.12) \times 10^{-2}$ & $(2.88 \pm 0.09) \times 10^{-2}$ & $(4.25 \pm 1.38) \times 10^{-2}$ \\
3.41 & $(5.05 \pm 0.11) \times 10^{-2}$ & $(2.61 \pm 0.08) \times 10^{-2}$ & $(3.82 \pm 1.20) \times 10^{-2}$ \\
7.98 & $(5.73 \pm 0.37) \times 10^{-3}$ & $(1.99 \pm 0.36) \times 10^{-3}$ & $(3.86 \pm 1.87) \times 10^{-3}$ \\
8.33 & $(6.32 \pm 0.34) \times 10^{-3}$ & $(2.59 \pm 0.26) \times 10^{-3}$ & $(4.46 \pm 1.86) \times 10^{-3}$ \\
9.72 & $(3.68 \pm 0.15) \times 10^{-3}$ & $(2.37 \pm 0.16) \times 10^{-3}$ & $(3.03 \pm 0.66) \times 10^{-3}$ \\
10.49 & $(3.67 \pm 0.16) \times 10^{-3}$ & $(2.17 \pm 0.18) \times 10^{-3}$ & $(2.92 \pm 0.75) \times 10^{-3}$ \\
\hline
\end{tabular}

has been smoothed to angular resolution 11.6 to match the ARCADE resolution. The data segregate into two tracks, an upper track from emission near the Galactic center and a lower track from emission closer to the Cygnus region. Both tracks show radio emission proportional to the square root of the $\mathrm{C}$ II intensity. This would be expected if the radio emission were dominated by synchrotron emission (proportional to the density $n$ in the interstellar medium), but with collisionally excited $\mathrm{C}$ II intensity proportional to $n^{2}$. Provided both the high-latitude radio continuum and the $\mathrm{C}$ II line intensity predominantly originate from a small number of emitting regions along each line of sight, the radio temperature $T$ and line intensity $I$ would then scale as $T \propto I^{\kappa}$ with $\kappa=1 / 2$. Emission along the same line of sight from a large number of regions with different local densities would force the relation closer to linear $(\kappa=1)$, as would a significant admixture of radio free-free emission (with antenna temperature proportional to $n^{2}$ ) The observed dependence shows $\kappa$ in the range $0.5-0.7$, as can be verified by the lack of significant curvature in Figure 4 . The absence of any such curvature provides additional evidence that free-free emission is faint compared to synchrotron at the ARCADE 2 bands. Note, though, that if a separate template is provided for the synchrotron component, the C II map may then be used to trace the fainter free-free component (at least at the ARCADE angular resolution).

The prominent segregation in Figure 4 results at least in part from the limited sky coverage. The brightest regions in the ARCADE 2 maps occur at the portion of the ARCADE 2 sky coverage closest to the Galactic center region and again at the portion closest to the Cygnus region. The ratio of $\mathrm{C}$ II intensity to the far-infrared continuum is known to differ by a factor of two between these regions (Fixsen et al. 1999). The radio/C II correlation for the ARCADE 2 sky coverage is dominated by two segregated tracks. Fitting a single slope to the ARCADE 2 data could induce a systematic error as small shifts in sky coverage view more of one track than the other. We minimize this effect by fitting two components to the radio/C II correlation plots,

$$
T_{A}(v, p)=\sum_{i=1}^{2} b_{i}(v)+a_{i}(v)\left(I_{C}(p)\right)^{0.5},
$$

where $T_{A}$ is the antenna temperature at frequency $\nu, I_{C}$ is the $\mathrm{C}$ II intensity, and $p$ denotes a pixel index. After a first guess at the fit parameters (intercept and slope for each line), we assign each point to one line or the other based on the difference in antenna temperature between that point and either line. We then recompute the fit parameters for each line using only those points assigned to that line, and then reassign all points based on the new fit parameters. Several iterations suffice to produce a stable solution independent of the initial guess. We then define the radio/C II correlation slope as the arithmetic mean of the two fitted components, $\langle a(v)\rangle=0.5\left[a_{1}(v)+a_{2}(v)\right]$, with uncertainty broad enough to bracket the two cases. The mean value thus derived is consistent with similar analysis fitting a single slope to the full sky but is less sensitive to small shifts in the sky coverage. Table 3 shows the radio/C II correlation.

The observed correlation between radio emission and $\mathrm{C}_{\text {II }}$ intensity allows an independent determination of the associated Galactic radio emission toward the Galactic poles,

$$
T_{G}(v)=\langle a(v)\rangle\left(I_{\text {cap }}\right)^{\kappa},
$$

where $I_{\text {cap }}$ is the mean intensity of the C II map at the polar caps (Galactic latitude $|b|>75^{\circ}$ ) and we take $\kappa=0.5$. Higher values of $\kappa$ within the observed range $0.5-0.7$ lower the estimated Galactic emission by less than the half the model uncertainty. Since the correlation coefficient $a(v)$ is derived using spatial fluctuations in the observed radio and line intensity, this method accounts at least roughly for both longitudinal as well as latitudinal structure on the sky. However, to the extent that the radio emission is not co-located with the line emission, the correlated emission does not uniquely determine the zero level of either component.

We repeat both the cosecant fit (Equation (5)) and the radio/C II correlation (Equation (7)) independently for the northern and southern hemispheres, using each of the ARCADE 2 sky maps as well as a selection of lower-frequency radio surveys. Surveys at $22 \mathrm{MHz}$ (Roger et al. 1999), $45 \mathrm{MHz}$ (Maeda et al. 1999; Alvarez et al. 1997), 408 MHz (Haslam et al. 1981), and $1420 \mathrm{MHz}$ (Reich et al. 2001; Reich \& Reich 1986) have full or nearly full-sky coverage at frequencies where the sky brightness is dominated by Galactic radio emission. For each sky map, we compute the cosecant slope $T_{G}(v)$ and its uncertainty, including both statistical and calibration uncertainties. Although the binned data are broadly consistent with a plane-parallel model, higher-order structure causes the binned data to show greater scatter about the best-fit slope than would be expected given the formal statistical uncertainty in the mean of each bin. We account for higher-order structure by inflating the statistical uncertainty to force $\chi^{2}$ to unity per degree of freedom about the best-fit line. We then add the calibration uncertainty (of order $10 \%$ for the low-frequency radio surveys) in 


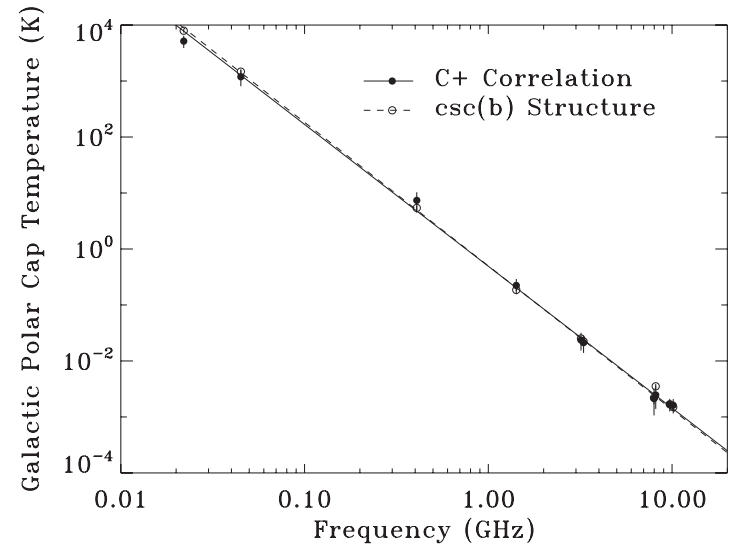

Figure 5. Galactic emission $T_{G}(v)$ toward the North galactic pole, derived from a cosecant fit to the spatial structure (open circles) and the radio/C II correlation (filled circles). Both methods agree and are consistent with a single power law over the frequency range $22 \mathrm{MHz}$ to $10 \mathrm{GHz}$. The fitted Galactic temperature toward the polar caps can be combined with the template model of the spatial structure to fully specify the Galactic model.

quadrature with the (inflated) statistical uncertainty to derive the total uncertainty in the cosecant slope for each sky survey.

Figure 5 compares the Galactic temperature toward the north polar cap, derived from mean $C_{\text {II }}$ correlation, to the value derived from the cosecant fit. Both methods provide similar estimates for the Galactic component of the total brightness temperature toward the north polar cap. This Galactic component is consistent with a single power law

$$
T_{G}(\nu)=T_{\mathrm{Gal}}\left(\nu / \nu_{0}\right)^{\beta}
$$

with spectral index $\beta=-2.55 \pm 0.03$ and amplitude $T_{\mathrm{Gal}}=$ $10.12 \pm 0.90 \mathrm{~K}$ at reference frequency $\nu_{0}=0.31 \mathrm{GHz} .{ }^{9}$

Several mechanisms could alter the total high-latitude Galactic emission from a power-law form. Energy losses from cosmic rays propagating in the interstellar medium will cause a progressive steepening of the synchrotron spectrum at higher frequencies. Comparison of the $408 \mathrm{MHz}$ survey with data from the WMAP mission shows some evidence for such steepening at frequencies above $20 \mathrm{GHz}$ (Gold et al. 2009; Bennett et al. 2003). Free-free absorption can become appreciable at sufficiently low frequencies, lowering the total temperature to cause a shallower spectrum below $\sim 50 \mathrm{MHz}$ where the synchrotron antenna temperature exceeds the typical $8000 \mathrm{~K}$ electron temperature in the diffuse interstellar medium. We may use the modeled Galactic temperatures to examine such effects.

The data in Figure 5 are not independent. Data from different ARCADE bands share common-mode thermometry uncertainty and small corrections for instrumental emission. The gain calibration of the $22 \mathrm{MHz}$ survey is derived in part from comparison of the $22 \mathrm{MHz}$ survey to the $408 \mathrm{MHz}$ survey, thereby correlating these results. We account for these correlated uncertainties when deriving the fitted parameters. Including these correlations, the distribution of the individual points in Figure 5 with respect to the best-fit power-law model is consistent with the measurement uncertainties. Additional free parameters (e.g., for possible spectral steepening) are not statistically justified.

The analysis above uses a single map ( $\mathrm{C}$ II line emission) to trace Galactic emission from the diffuse interstellar medium.

\footnotetext{
9 We chose reference frequency $0.31 \mathrm{GHz}$ to minimize the covariance between the fitted amplitude and spectral index. See the discussion in Fixsen et al. (2011).
}

Table 4

Galactic Emission ${ }^{\mathrm{a}}$ along Selected Lines of Sight

\begin{tabular}{lcrrc}
\hline \hline Parameter & Technique & \multicolumn{1}{c}{ North Polar Cap } & South Polar Cap & Coldest Patch \\
\hline \multirow{4}{*}{$T_{\text {Gal }}(\mathrm{K})$} & Radio/C II & $9.69 \pm 2.14$ & $8.83 \pm 2.07$ & $5.40 \pm 3.86$ \\
& $\csc |b|$ & $10.21 \pm 0.99$ & $8.29 \pm 0.90$ & $\ldots$ \\
& Weighted mean & $10.12 \pm 0.90$ & $8.38 \pm 0.83$ & $5.40 \pm 3.86$ \\
\hline \multirow{3}{*}{$\beta$} & Radio/C II & $-2.52 \pm 0.07$ & $-2.53 \pm 0.07$ & $-2.52 \pm 0.11$ \\
& $\csc |b|$ & $-2.55 \pm 0.04$ & $-2.64 \pm 0.05$ & $\ldots$ \\
& Weighted mean & $-2.55 \pm 0.03$ & $-2.63 \pm 0.03$ & $-2.52 \pm 0.11$ \\
\hline
\end{tabular}

Note. ${ }^{\text {a }}$ Galactic emission $T_{G}(v)=T_{\mathrm{Gal}}\left(v / v_{0}\right)^{\beta}$ with reference frequency $v_{0}=$ $0.31 \mathrm{GHz}$

If a significant fraction of Galactic radio emission originated from a component of the interstellar medium not well sampled by $\mathrm{C}$ II emission, the resulting correlation would underestimate the total Galactic emission toward the polar cap. We test for additional microwave emission from other components of the interstellar medium by repeating the correlation analysis using a simultaneous fit to three line maps chosen to sample different components of the diffuse interstellar medium: the (square root of the) $C O B E$ /FIRAS map of $\mathrm{C}$ II emission, a map of $\mathrm{H} \alpha$ emission (Finkbeiner 2003), and the Leiden/Argentine/Bonn Galactic H I Survey (Kalberla et al. 2005). The simultaneous fit produces results nearly identical to a fit using just the C II map, shifting the derived Galactic polar cap temperature by $0.2 \pm$ $0.4 \mathrm{~K}$ at reference frequency $0.31 \mathrm{GHz}$. Since the limited ARCADE 2 sky coverage increases the effect of covariance between the different line maps, we use the results derived from the single $\mathrm{C}$ II correlation.

\subsection{Composite Galactic Model}

Removing Galactic emission from the ARCADE 2 data requires a model for both the spatial structure and the total Galactic emission. At each ARCADE frequency, we generate a full-sky model of the spatial structure using full-sky template maps multiplied by the coefficients fitted within the region observed by ARCADE 2 (Table 2). We then add a constant to the template model to match the total Galactic emission derived from the C II and csc $|b|$ fits toward selected reference positions.

We independently model the total Galactic emission toward the north and south Galactic polar caps $\left(|b|>75^{\circ}\right)$. For each reference position, we compute the total Galactic emission associated with either the plane-parallel structure (Equation (5)) or the C II emission (Equation (7)). We apply each method to the ARCADE 2 and radio surveys, and parameterize the resulting multi-frequency results using a power-law model (Equation (8)). As a cross-check, we also estimate the total Galactic emission for the coldest patch in the Northern hemisphere, consisting of all pixels within $15^{\circ}$ of $b=48^{\circ}, l=196^{\circ}$ (north of the Galactic anti-center). Since by definition a mid-latitude cold spot is inconsistent with a plane-parallel structure, the estimate for this position is based only on the radio/C II correlation. Table 4 shows the estimated Galactic normalization $T_{\mathrm{Gal}}$ and spectral index $\beta$ for each reference position. We obtain similar results using either the spatial morphology or the radio/C II correlation.

We fix the offset of the template model (Equation (3)) by computing the temperature of the template model toward these same regions, then adding a constant $\alpha_{0}(\nu)$ to the template model at each frequency $v$ to force the template model to match the power-law model along these lines of sight. The resulting composite model of Galactic emission can be used 
to correct the calibrated time-ordered data in order to estimate the $\mathrm{CMB}$ monopole temperature and the extragalactic radio background (Fixsen et al. 2011). The offsets derived from the three independent regions agree within $5 \mathrm{mK}$ at $3 \mathrm{GHz}$, which we adopt as the uncertainty in the zero level of composite Galactic model.

The spectral index derived for the Galactic emission toward the polar caps or coldest region, $\beta \approx-2.57 \pm 0.03$, is consistent with the spectral index $\beta=-2.5 \pm 0.1$ derived from the synchrotron template fit only to ARCADE 2 data, indicating that high-latitude Galactic emission is dominated by synchrotron emission at frequencies below $10 \mathrm{GHz}$. A synchrotron spectral index of -2.57 is significantly flatter than the values derived from measurements at higher frequencies, but is consistent with the majority of measurements below $10 \mathrm{GHz}$ (see, e.g., the tabulation in Rogers \& Bowman 2008). Notable exceptions are the measurements by Tartari et al. (2008) and Platania et al. (1998), which prefer $\beta \approx-2.8$ for the synchrotron component. Both of these results are derived from a single strip at declination $\delta=+42^{\circ}$ and may not be representative of larger regions of the sky.

Although a plane-parallel model provides a good description of the latitudinal dependence of the radio sky, the projected polar cap temperatures from a csc $|b|$ model are well below the actual measured values. For example, the North Galactic pole has measured temperature $19 \pm 3 \mathrm{~K}$ at $408 \mathrm{MHz}$, while a $\csc |b|$ fit to the same $408 \mathrm{MHz}$ map predicts a polar contribution of only $5.1 \pm 0.6 \mathrm{~K}$. Similar results apply to the Southern hemisphere, where the measured polar cap temperature of $21 \pm 3 \mathrm{~K}$ significantly exceeds the value $4.0 \pm 0.5 \mathrm{~K}$ obtained from a csc $|b|$ fit. Only $2.7 \mathrm{~K}$ of the difference can be attributed to emission from the $\mathrm{CMB}$, leaving a large $(12 \mathrm{~K})$ residual. Several authors have noted the existence of this bright, isotropic component of high-latitude radio emission (Phillips et al. 1981; Beuermann et al. 1985; Sun et al. 2008; Everett et al. 2010). The Galactic model presented here is consistent with these models. Radio emission at latitude $b>40^{\circ}$ is dominated by an emission component not associated with the plane-parallel structure of the Galaxy, but residing in either a Galactic halo or an isotropic extragalactic background.

\section{SPINNING DUST}

The ARCADE 2 data can also be used to search for additional microwave emission associated with interstellar dust. The microwave sky is known to contain a component spatially correlated with far-infrared dust emission but not with synchrotrondominated surveys at 408 or $1420 \mathrm{MHz}$ (Kogut et al. 1996a, 1996b; de Oliveira-Costa et al. 1997; Leitch et al. 1997; Bennett et al. 2003). The frequency spectrum of the correlated component differs markedly from the emission spectrum of thermal dust, showing a spectral index $\beta \approx-2.2$ in antenna temperature from 20 to $50 \mathrm{GHz}$.

Two main candidates have emerged to explain this "anomalous" correlated emission component. Electric dipole emission from a population of small, rapidly rotating dust grains will produce a spectrum with a broad peak in the frequency range 20-40 GHz (Draine \& Lazarian 1998). Miville-Deschênes et al. (2008) analyze data from the WMAP and conclude that spinning dust accounts for the majority of emission in the Galactic plane at $23 \mathrm{GHz}$. An alternative model explains the correlated emission as flat-spectrum synchrotron emission associated with star formation activity (Bennett et al. 2003; Gold et al. 2009). Energy losses as electrons propagate from their origin steepen the syn- chrotron index away from these sources. Since a flat-spectrum component will increasingly dominate at higher frequencies, the spatial morphology of synchrotron emission at higher frequencies should increasingly resemble thermal dust emission from the same star formation activity.

Efforts to distinguish between these models have largely focused on the frequency spectrum of the dust-correlated component inferred by correlating a map tracing thermal dust emission against microwave maps at different observing frequencies. The correlation with thermal dust emission is seen to peak at frequencies between 20 and $30 \mathrm{GHz}$, falling at frequencies below $23 \mathrm{GHz}$ (de Oliveira-Costa et al. 2004; Fernández-Cerezo et al. 2006; Hildebrandt et al. 2007). This falling spectrum at low frequencies has been cited as evidence favoring spinning dust models.

The decrease in dust-correlated emission at frequencies below $20 \mathrm{GHz}$, although consistent with spinning dust emission, does not necessarily support such models over the flat-spectrum synchrotron alternative. Both models predict weaker correlation with thermal dust emission at frequencies below $20 \mathrm{GHz}$. For the spinning dust model, the weaker correlation results from the lower amplitude of the spinning dust emission. The flatspectrum synchrotron model, however, also predicts a weaker correlation at lower frequencies, since by construction the spatial morphology of the sky in this model varies smoothly from the WMAP data at $23 \mathrm{GHz}$ (observed to correlate well with the thermal dust morphology) to the Haslam map at $408 \mathrm{MHz}$ (observed to correlate only weakly with thermal dust emission). Model discrimination based on cross-correlations with thermal dust emission must rely on rigorous statistical comparison of competing models, and not simply on the general trend toward weaker correlations at lower frequencies.

A simpler test relies on the absolute spectrum of Galactic emission. At frequencies below $30 \mathrm{GHz}$, thermal dust emission is negligible, so that the total Galactic emission becomes a superposition of free-free, synchrotron, and spinning dust emission. Both free-free and synchrotron emission increase monotonically at lower frequencies. Spinning dust, in contrast, decreases in amplitude below $20 \mathrm{GHz}$, so that the spectrum of the combined Galactic emission below $20 \mathrm{GHz}$ can be used to place limits on the contribution of spinning dust without reference to detailed spatial correlations with a thermal dust template.

Figure 6 illustrates the concept. Consider Galactic emission consisting of a superposition of synchrotron and spinning dust (ignoring for the moment the smaller contribution from free-free emission). Following Miville-Deschênes et al. (2008), we specify the spinning dust amplitude as a fraction of the total Galactic brightness at $23 \mathrm{GHz}$, assumed here to represent the peak in the dust spectrum. As the dust normalization is increased, the synchrotron amplitude at $23 \mathrm{GHz}$ must decrease to keep the total emission constant. The spinning dust spectrum falls rapidly, so that emission below $10 \mathrm{GHz}$ is dominated by the synchrotron component. A model with high dust normalization (red curves in Figure 6) will thus have fainter synchrotron emission at frequencies of a few $\mathrm{GHz}$, while a model with less spinning dust (blue curves) will have brighter synchrotron emission. This shift in the amplitude of Galactic emission at frequencies below $10 \mathrm{GHz}$ is a sensitive test for spinning dust, without resort to detailed spatial correlations.

We implement this test using a simple model of Galactic emission. We assume that the spatial distribution of spinning dust emission is traced by a template map of thermal dust 


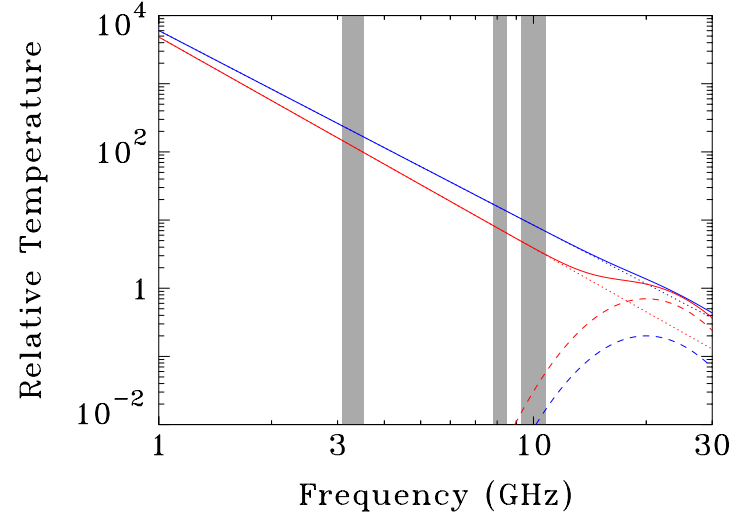

Figure 6. Synchrotron and spinning dust emission for two models with different spinning dust amplitude. The red (blue) curves show emission from a model with high (low) dust normalization. Dashed lines show the dust emission, dotted lines show synchrotron emission, and solid lines show the combined emission from each model. The amplitude of the combined emission is fixed at both $23 \mathrm{GHz}$ and $408 \mathrm{MHz}$. Measurements below $10 \mathrm{GHz}$ are dominated by synchrotron, which can be used to infer the dust normalization: models with more dust emission at $23 \mathrm{GHz}$ have lower synchrotron emission. Gray bars indicate the ARCADE 2 frequency bands.

(A color version of this figure is available in the online journal.)

emission, and fix the amplitude of associated spinning dust emission by scaling the template map so that the rescaled dust map forms a specified fraction of the Galactic plane intensity measured by the WMAP $23 \mathrm{GHz}$ map. This allows a simple normalization of spinning dust in terms of its relative contribution to the total Galactic plane intensity at $23 \mathrm{GHz}$. We use the $C O B E / \mathrm{DIRBE} 240 \mu \mathrm{m}$ map of thermal dust emission (Reach et al. 1996) as the thermal dust template since it is dominated by thermal dust emission but unaffected by extinction in the plane. For specificity, we define the Galactic plane mask using all pixels lying within the ARCADE 2 observation pattern with latitude $|b|<20^{\circ}$, and use this mask for all computations so that the model results are not affected by differing sky coverage between ARCADE 2 and other surveys.

We then model the Galactic emission spectrum as follows. We first correct the WMAP $23 \mathrm{GHz}$ map and the Haslam $408 \mathrm{MHz}$ map by subtracting the WMAP maximum entropy model of free-free emission (Gold et al. 2009) using a spectral index $\beta_{\mathrm{ff}}=-2.15$. The corrected maps then contain only synchrotron and (possibly) spinning dust emission. We assume that the spinning dust spectrum peaks at $23 \mathrm{GHz}$ and scale the normalized spinning dust map to lower frequencies using the Draine \& Lazarian (1998) model for the warm neutral medium. After correction for free-free and spinning dust emission, the $23 \mathrm{GHz}$ and $408 \mathrm{MHz}$ maps contain only synchrotron emission, which we use to define the synchrotron amplitude and spectral index for each pixel.

The resulting model (synchrotron, free-free, and spinning dust) can be used to estimate the combined Galactic emission at frequencies between $408 \mathrm{MHz}$ and $23 \mathrm{GHz}$. We compare the model to the ARCADE 2 data by smoothing the model map of combined emission to the ARCADE 2 angular resolution, and then computing the mean Galactic emission of the smoothed model for all pixels within the ARCADE 2 galactic plane mask. Figure 7 shows the result. If spinning dust is negligible, the synchrotron contribution at $23 \mathrm{GHz}$ is maximal and the model approximates the flat-spectrum synchrotron model. As the spinning dust amplitude increases, the synchrotron contribution at $23 \mathrm{GHz}$ decreases and the synchrotron spectral index steepens.

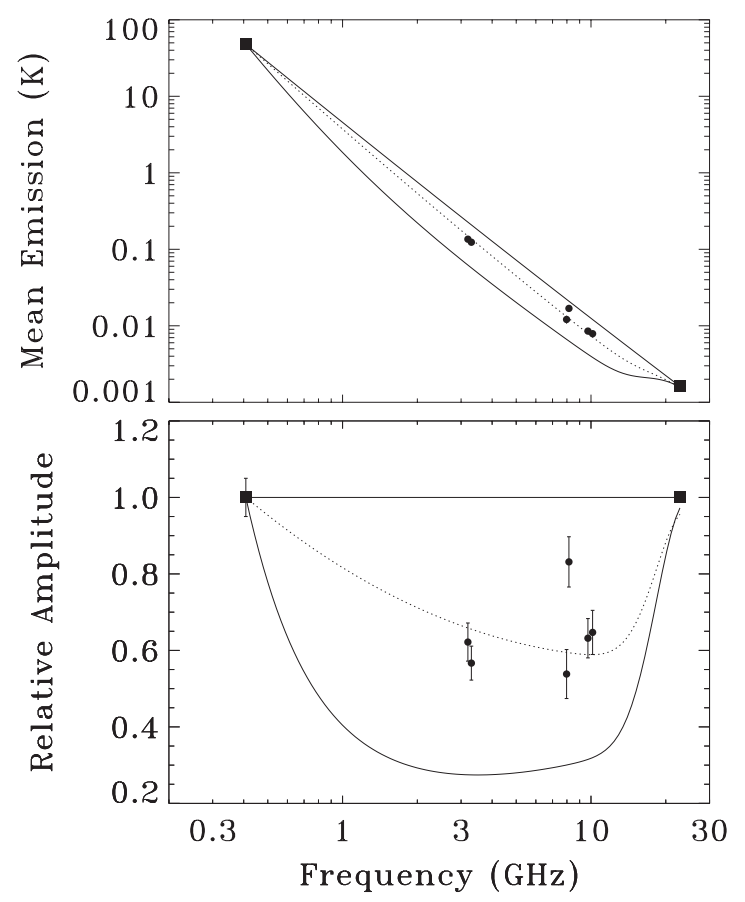

Figure 7. Mean intensity of Galactic plane emission for the ARCADE 2 data, compared to model predictions with and without spinning dust (see the text). Top panel: model predictions and data. The upper solid curve shows the model prediction for no spinning dust, averaged over the ARCADE 2 sky coverage with $|b|<20^{\circ}$. The lower solid curve shows the model prediction for spinning dust amplitude equal to $60 \%$ of the total Galactic plane emission at $23 \mathrm{GHz}$. The dotted curve shows the best fit to the ARCADE 2 data. The spectrum of emission from spinning dust is assumed to peak at $23 \mathrm{GHz}$, so a higher spinning dust fraction at $23 \mathrm{GHz}$ produces lower total emission at the ARCADE 2 frequencies. Bottom panel: the same data, normalized by dividing each point by the model prediction for no spinning dust. Galactic plane emission observed by ARCADE 2 is consistently fainter than expected for a model with no spinning dust and is consistent with spinning dust contributing $0.4 \pm 0.1$ of the total Galactic plane emission at reference frequency $23 \mathrm{GHz}$.

The combination of lower synchrotron amplitude and falling dust spectrum combine to lower the total model emission across the ARCADE 2 frequency bands. The ARCADE 2 data lie below the model prediction for no spinning dust and are consistent with spinning dust contributing $0.4 \pm 0.1$ of the total $K$-band Galactic plane emission.

WMAP is a differential instrument and is insensitive to any monopole emission component. The zero level of the $23 \mathrm{GHz}$ map is set using the cosecant dependence on Galactic latitude (Hinshaw et al. 2009). The $408 \mathrm{MHz}$ survey and the ARCADE 2 data, however, both include monopole contributions. To prevent discrepant treatment of the map zero levels from affecting the model predictions, we remove a monopole from the $408 \mathrm{MHz}$ survey and the ARCADE 2 sky maps, and reset the zero level of each map using a csc $|b|$ fit to the spatial structure in each map to match the processing of the WMAP data. We restrict analysis to pixels at low Galactic latitude $\left(|b|<20^{\circ}\right)$ where Galactic emission is brightest so that uncertainties in the zero level have minimal effect. The largest uncertainty in the analysis is the correction for free-free emission. We use the WMAP maximum entropy model of free-free emission, derived assuming that spinning dust contributes negligibly to the total Galactic emission. We test the sensitivity of the result to the free-free normalization by repeating the analysis using the same maximum entropy model scaled by a constant normalization factor. Changing the free-free normalization by a factor of two 
affects the best-fit spinning dust normalization by approximately 0.08 and is included in the total quoted uncertainty.

\section{CONCLUSIONS}

We use the ARCADE 2 absolutely calibrated observations of the sky to model Galactic emission at frequencies 3, 8, and $10 \mathrm{GHz}$ and angular resolution 11.6. $T-T$ plots of the binned sky maps show net spectral index $\beta=-2.43 \pm 0.03$ between 3.3 and $8.3 \mathrm{GHz}$ and $\beta=-2.47 \pm 0.02$ between 3.3 and $10.3 \mathrm{GHz}$, consistent with a superposition of synchrotron and free-free emission. The spatial structure in the maps can be described using two spatial templates, the Haslam $408 \mathrm{MHz}$ survey to trace synchrotron emission and the $C O B E$ /FIRAS map of $\mathrm{C}_{\text {II }}$ emission to trace free-free emission. Fitting these templates to the ARCADE 2 maps yields spectral index $\beta_{\text {synch }}=-2.5 \pm 0.1$ for emission traced by the synchrotron template, with free-free normalization $\left\langle T_{\mathrm{ff}} / T_{\text {total }}\right\rangle=0.10 \pm 0.01$ evaluated for latitudes $|b|<10^{\circ}$ in the lowest ARCADE 2 channel at $3.15 \mathrm{GHz}$.

The template model only specifies Galactic emission up to an additive constant. We fully specify the Galactic model by computing the temperature of the template model toward the north and south polar caps, then adding a constant to the template model to match the Galactic temperature derived from a simple plane-parallel model. We extend the analysis to full-sky surveys at lower frequencies and find that Galactic polar cap temperature is consistent with a single power law over the frequency range $22 \mathrm{MHz}$ to $10 \mathrm{GHz}$, with spectral index $\beta=-2.55 \pm 0.03$ and normalization $10.12 \pm 0.90 \mathrm{~K}$ at reference frequency $v_{0}=0.31 \mathrm{GHz}$. Emission associated with the plane-parallel structure accounts for only $30 \%$ of the observed high-latitude sky temperature, with the residual in either a Galactic halo or an isotropic extragalactic background.

ARCADE2 produces well-calibrated maps of Galactic emission. We use these maps to test for contributions from spinning dust near the Galactic plane. Previous tests for spinning dust at frequencies below $20 \mathrm{GHz}$ have used only the spatial correlation with template maps tracing thermal dust emission. The frequency dependence of the dust-correlated component is not a stringent test for spinning dust, since a weaker correlation at lower frequencies is expected both for models with significant spinning dust contributions as well as models with flat-spectrum synchrotron but no spinning dust. We use a simple model of the total Galactic emission to predict the mean intensity of the Galactic plane in the region observed by ARCADE 2. The model normalizes the spinning dust contribution at $23 \mathrm{GHz}$, and computes the expected total contribution from free-free, synchrotron, and spinning dust as a function of frequency and spinning dust normalization. The ARCADE 2 data consistently show less emission in the Galactic plane than a model with no spinning dust and are consistent with spinning dust contributing $0.4 \pm 0.1$ of the Galactic plane emission at $23 \mathrm{GHz}$.

We thank the staff of the Columbia Scientific Balloon Facility for their capable support throughout the integration, launch, flight, and recovery of the ARCADE 2 mission. We thank the students whose work helped make ARCADE 2 possible: Adam Bushmaker, Jane Cornett, Sarah Fixsen, Luke Lowe, and Alexander Rischard. We thank P. Reich for providing the $45 \mathrm{MHz}$ and $1420 \mathrm{MHz}$ surveys in electronic format. We acknowledge use of the HEALPix software package (Górski et al. 2005). This research is based upon work supported by the
National Aeronautics and Space Administration through the Science Mission Directorate under the Astronomy and Physics Research and Analysis suborbital program. The research described in this paper was performed in part at the Jet Propulsion Laboratory, California Institute of Technology, under a contract with the National Aeronautics and Space Administration. T.V. acknowledges support from CNPq grants 466184/00-0, 305219-2004-9, and 303637/2007-2-FA, and the technical support from Luiz Reitano. C.A.W. acknowledges support from CNPq grant 307433/2004-8-FA.

\section{REFERENCES}

Alvarez, H., Aparici, J., May, J., \& Olmos, F. 1997, A\&AS, 124, 315

Bennett, C. L., et al. 2003, ApJS, 148, 97

Beuermann, K., Kanblach, G., \& Berkhuijsen, E. M. 1985, A\&A, 153, 17 de Oliveira-Costa, A., Kogut, A., Devlin, M. J., Netterfield, C. B., Page, L. A., \& Wollack, E. J. 1997, ApJ, 482, L17

de Oliveira-Costa, A., Tegmark, M., Davies, R. D., Gutiérrez, C. M., Lasenby, A. N., Rebolo, R., \& Watson, R. A. 2004, ApJ, 606, L89

Dobler, G., \& Finkbeiner, D. P. 2008, ApJ, 680, 1222

Draine, B. T., \& Lazarian, A. 1998, ApJ, 494, L19

Everett, J. E., Schiller, Q. G., \& Zweibel, E. G. 2010, ApJ, 711, 13

Fernández-Cerezo, S., et al. 2006, MNRAS, 370, 15

Finkbeiner, D. P. 2003, ApJS, 146, 407

Finkbeiner, D. P., Davis, M., \& Schlegel, D. J. 1999, ApJ, 524, 867

Fixsen, D. J., Bennett, C. L., \& Mather, J. C. 1999, ApJ, 526, 207

Fixsen, D. J., Kogut, A., Levin, S., Limon, M., Lubin, P., Mirel, P., Seiffert, M., \& Wollack, E. 2004, ApJ, 612, 86

Fixsen, D. J., et al. 2011, ApJ, 734, 5

Gold, B., et al. 2009, ApJS, 180, 265

Górski, K. M., Hivon, E., Banday, A. J., Wandelt, B. D., Hansen, F. K., Reinecke, M., \& Bartelmann, M. 2005, ApJ, 622, 759

Haslam, C. G. T., Klein, U., Salter, C. J., Stoffel, H., Wilson, W. E., Cleary, M. N., Cooke, D. J., \& Thomasson, P. 1981, A\&A, 100, 209

Hildebrandt, S. R., Rebolo, R., Rubiño-Martín, J. A., Watson, R. A., Gutiérrez, C. M., Hoyland, R. J., \& Battistelli, E. S. 2007, MNRAS, 382, 594

Hinshaw, G., et al. 2009, ApJS, 180, 225

Kalberla, P. M. W., Burton, W. B., Hartmann, D., Arnal, E. M., Bajaja, E., Morras, R., \& Pöppel, W. G. L. 2005, A\&A, 440, 775

Kogut, A., Banday, A. J., Bennett, C. L., Gorski, K. M., Hinshaw, G., \& Reach, W. T. 1996a, ApJ, 460, 1

Kogut, A., Banday, A. J., Bennett, C. L., Gorski, K. M., Hinshaw, G., Smoot, G. F., \& Wright, E. I. 1996b, ApJ, 464, L5

Kogut, A., Fixsen, D. J., Levin, S., Limon, M., Lubin, P. M., Mirel, P., Seiffert, M., \& Wollack, E. 2004, ApJS, 154, 493

Leitch, E. M., Readhead, A. C. S., Pearson, T. J., \& Myers, S. T. 1997, ApJ, 486, L23

Maeda, K., Alvarez, H., Aparici, J., May, J., \& Reich, P. 1999, A\&AS, 140, 145

Miville-Deschênes, M.-A., Ysard, N., Lavabre, A., Ponthieu, N., Macías-Pérez, J. F., Aumont, J., \& Bernard, J. P. 2008, A\&A, 490, 1093

Phillips, S., Kearsey, S., Osborne, J. L., Haslam, C. G. T., \& Stoffel, H. 1981, A\&A, 103, 405

Platania, P., Bensadoun, M., Bersanelli, M., de Amici, G., Kogut, A., Levin, S., Maino, D., \& Smoot, G. F. 1998, ApJ, 505, 473

Reach, W. T., Franz, B. A., Kelsall, T., \& Weiland, J. L. 1996, in AIP Conf. Proc. 348, The COBE Workshop: Unveiling the Cosmic Infrared Background, ed. E. Dwek (Melville, NY: AIP), 37

Reich, P., \& Reich, W. 1986, A\&AS, 63, 205

Reich, P., Testori, J. C., \& Reich, W. 2001, A\&A, 376, 861

Roger, R. S., Costain, C. H., Landecker, T. L., \& Swerdlyk, C. M. 1999, A\&AS, 137,7

Rogers, A. E., \& Bowman, J. D. 2008, AJ, 136, 641

Rybicki, G. B., \& Lightman, A. 1979, Radiative Processes in Astrophysics (New York: Wiley)

Seiffert, M., et al. 2011, ApJ, 734, 6

Singal, J., Wollack, E., Kogut, A., Limon, M., Mirel, P., Lubin, P., \& Seiffert, M. 2005, Rev. Sci. Inst., 76, 124703

Singal, J., et al. 2006, ApJ, 653, 835

Singal, J., et al. 2011, ApJ, 730, 138

Sun, X. H., Reich, W., Waelkens, A., \& Enßlin, T. A. 2008, A\&A, 477, 573

Tartari, A., Zannoni, M., Gervasi, M., Boella, G., \& Sironi, G. 2008, ApJ, 688, 32 